Continued follow-up as an outpatient has shown no evidence of further urinary infection, and liver-function tests nine months later showed serum bilirubin $0.5 \mathrm{mg} . / 100 \mathrm{ml}$., thymol turbidity 3 units, zinc sulphate 4 units, with a moderately elevated alkaline phosphatase of 34 K.A. units.

\section{Discussion}

The association between jaundice and urinary infection has previously been documented. Görter and Lignac (1928) described the cases of three infants with pyelitis complicated by jaundice, the one fatal case having necropsy evidence suggestive of pyaemia. Several series have been published since, notably those of Bernstein and Brown (1962) and Hamilton and SassKortsak (1963). In these series the emphasis has been on jaundice as a feature of septicaemia associated with pyelitisbeing primarily studies of infants in whom blood culture was positive.

Bernstein and Brown (1962) analysed 70 cases of infants with positive blood cultures (ante mortem or post mortem), all of whom came to necropsy. Of these, $14(20 \%)$ were jaundiced and seven of the nine who presented with jaundice after the first week of life had evidence of pyelonephritis-five due to $E$. coli and two to paracolon bacilli. Six of the seven cases were jaundiced when first seen, jaundice being terminal in the seventh. The common histological lesions were bile stasis, intracellular pigment retention, and " toxic" cellular abnormalities. The authors felt that, in the absence of evidence of mechanical ductal obstruction, jaundice was due to inability of partially injured cells to excrete bile. Incidentally, none of the seven cases with pyelonephritis had underlying malformations of the genitourinary tract.

The series described by Hamilton and Sass-Kortsak (1963) consisted of 24 infants with septicaemia complicated by jaundice, which was the presenting symptom in 11 of the 24 cases. Among 18 infants in whom urine culture was performed 16 had urinary infections (13 due to $E$. coli) associated with gross pyuria. The nature of the jaundice was ill-defined, there being no evidence of actual infection of liver tissue in fatal cases coming to necropsy, and the authors felt that jaundice was initially due to red-cell haemolysis (Thurman, 1960), and, later, the result of toxic changes with hepatocellular dysfunction. Seven fatalities occurred in eight cases presenting in the first week of life, contrasting with four deaths among 16 cases presenting from the second week onwards. In a similar study of sepsis of obscure origin in the newborn Silverman and Homan (1949) reported jaundice as a feature in 14 out of 25 infants having positive blood cultures, pyuria being present in 9 of the 14 cases.

In our cases blood cultures were not undertaken. None of the infants had umbilical sepsis-a possible source of ascending infection leading to septic hepatitis or descending infection via the umbilical arteries leading to urinary infection-nor any signs of overt sepsis elsewhere. The mechanism whereby bacterial infection causes jaundice remains obscure. The low haemoglobin levels $(78 \%, 60 \%$, and $54 \%$ respectively in infants aged 3,4 , and 7 weeks) in the absence of overt loss of blood are suggestive of a haemolytic process. Absence of urobilinogen from the urine in Cases 1 and 2 is in keeping with an obstructive element which is almost certainly intrahepatic in origin, due to cellular injury. Zuelzer and Brown (1961) have postulated that a further factor in infection causing hyperbilirubinaemia, involving the immature liver, may be interference with the enzyme systems essential for conjugation of bilirubin.

The important feature of the cases described was the association of urinary infection with otherwise unexplained jaundice as the presenting symptom, the jaundice clearing with the cure of the infection. We would certainly support Hamilton and Sass-Kortsak (1963) in suggesting that any jaundice of sudden onset accompanied by leucocytosis raises suspicion of infection. In all cases of obscure jaundice in infancy urine culture is mandatory and blood culture desirable.

\section{Summary}

Jaundice was the presenting feature in four cases of urinary infection. The association is not uncommon, and its importance is stressed. Treatment of the urinary infection in each case resulted in disappearance of the jaundice.

\section{REFERENCES}

Bernstein, J., and Brown, A. K. (1962). Pediatrics, 29, 873.

Görter, E., and Lignac, G. O. E. (1928). Arch. Dis. Childh., 3, 232

Hamilton, J. R., and Sass-Kortsak, A. (1963). F. Pediat., 63, 121.

Silverman, W. A., and Homan. W. E. (1949). Pediatrics, 3, 157.

Thurman, W. G. (1960). Amer. F. Dis. Child., 100, 639 (Abstr.).

Zuelzer, W. W., and Brown, A. K. (1961). Ibid., 101, 87.

\title{
Squamous Carcinoma of the Thoracic Oesophagus in Malabsorption Syndrome
}

\author{
J. T. WRIGHT,* D.M., F.R.C.P.; P. C. RICHARDSON, † B.SC., M.R.C.P.
}

This paper reports the occurrence of carcinoma of the oesophagus in four patients with malabsorption syndrome. In these cases the growth was situated in the lower two-thirds of the oesophagus, and anaemia was megaloblastic in three and normocytic in one. These features differ from the classical Patterson-Kelly association of postcricoid lesions and irondeficiency anaemia.

The first case occurred in a man of 23 , and his death was the only one recorded for oesophageal carcinoma in the age group (20-24) in England and Wales during the year 1957. Inevitably there appeared to be a connexion between his lifelong

\footnotetext{
- Consultant Physician, the London Hospital, London E.1 ; Consultant
} Physician, Whipps Cross Hospital, London E.11.

t Senior Medical Registrar, the London Hospital, London E.1. history of coeliac disease and the development of oesophageal carcinoma, particularly as the second case, admittedly in an older patient, occurred in this hospital during the same year, but it seemed wise to await further cases before reporting the association. The third and fourth cases occurred in 1962 and 1964 respectively, and it was the latter one; also in a comparatively young person, that determined this report.

\section{Case 1}

This patient first attended the London Hospital in July 1952, at the age of 18. There was a story of diarrhoea since infancy with stunted growth noted from the age of 5 . He had had severe discomfort from recurrent aphthous stomatitis for as long as he could remember and the second dentition was poor. His height was $4 \mathrm{ft}$. 
$9 \frac{1}{2}$ in. $(146 \mathrm{~cm}$.) and he weighed $74 \mathrm{lb}$. $(33.6 \mathrm{~kg}$.). There were no secondary sex characters. His haemoglobin was $10.4 \mathrm{~g} . / 100 \mathrm{ml}$. and macrocytes were present in the peripheral blood.

Four months later, when he eventually agreed to come into hospital, his blood picture was unchanged. Two successive fat balances over three days on a diet containing $50 \mathrm{~g}$. fat/day, showed an average daily excretion of 7-8 g. fat. A follow-through bariummeal examination showed a small-intestinal malabsorption pattern, but nothing to suggest a structural lesion.

A gluten-free diet and folic acid 20 mg. t.d.s. led to rapid improvement, and six months after discharge his height was $5 \mathrm{ft} .3 \frac{1}{4}$ in. $(160.5 \mathrm{~cm}$.), weight $99 \mathrm{lb}$. (44.9 kg.), and haemoglobin $16.6 \mathrm{~g} . / 100$ $\mathrm{ml}$. The improvement continued until May, 1956, when he first noticed dysphagia for solids. Three months later oesophagoscopy revealed a squamous carcinoma $28 \mathrm{~cm}$. from the incisor teeth (just below the aortic arch). Oesophagectomy was successful, but the patient died after a massive haematemesis five months later.

Necropsy showed that the haemorrhage resulted from ulcerated metastases in the stomach. The jejunal mucosa was atrophic. There was widespread carcinomatosis.

\section{Case 2}

A married carpenter first attended the London Hospital in 1944, aged 37, with a five-year history of epileptic fits. He had suffered a fractured skull in a motor accident in 1935. The fits had been frequent, and during them he sustained many fractures. The fits were controlled by phenobarbitone and phenytoin.

Anaemia was diagnosed in 1946 (Hb 9.5 g./100 ml., M.C.H.C. $28.5 \%$ ). After six weeks' treatment with oral iron and ascorbic acid and twice-weekly injections of liver extract his haemoglobin rose to only $10 \mathrm{~g} . / 100 \mathrm{ml}$., but slow improvement followed and in 1949 his haemoglobin was $14 \mathrm{~g} . / 100 \mathrm{ml}$.

In 1954 he developed diarrhoea with pale bulky stools and began to lose weight rapidly. At this time he recalled that intermittent bouts of similar diarrhoea had occurred ever since his accident in 1935. He smoked 10 cigarettes a day, but did not drink alcohol.

On examination he was a small, pale, grey-haired, hunchbacked man with gross skeletal deformity, slightly clubbed fingernails which were of normal texture, and a smooth red tongue. Haemoglobin 11.2 g. $/ 100$ ml., M.C.H.C. $30 \%$, sternal marrow normoblastic, serum calcium $7.6 \mathrm{mg} . / 100 \mathrm{ml}$., phosphorus $3.8 \mathrm{mg} . / 100 \mathrm{ml}$., alkaline phosphatase 18.4 units. Barium-meal examination showed a steaturrhoea pattern in the small intestine. Fat absorption was 82 and $86 \%$ in two three-day studies. Radiographs showed pseudofractures in the left ulna and both right forearm bones in addition to gross deformities of spine and pelvis.

He responded well to gluten-free diet with supplements of folic acid, iron, calcium, and calciferol. He also received weekly intramuscular injections of liver extract, and six months later his haemoglobin was 14.2 g. $/ 100 \mathrm{ml}$. and serum calcium $9 \mathrm{mg} . / 100 \mathrm{ml}$.

Dysphagia developed in August 1957. Barium swallow showed a typical mid-oesophageal carcinoma extending from just below the aortic arch to 1 in. $(2.5 \mathrm{~cm}$.) above the cardia. Biopsy was impossible because of his thoracic deformity. Temporary clinical and radiological improvement resulted from radiotherapy, but the patient died at home in February 1958 at the age of 52. Post-mortem examination was not performed.

\section{Case 3}

A flourmill-roller man in 1949, aged 53, underwent total gastrectomy with Roux-en-Y anastomosis at the London Hospital for pyloric carcinoma. Recovery was complete and he returned to full employment.

In 1954 he presented with loss of weight, diarrhoea of recent onset, and severe megaloblastic anaemia confirmed by sternal marrow examination ( $\mathrm{Hb} 3.3 \mathrm{~g} . / 100 \mathrm{ml}$.). For many years he had poorly fitting dentures; he disliked greens, and his diet consisted mainly of meat and potatoes. He smoked 10-15 cigarettes a day, but never drank alcohol.

His faecal fat excretion was $12 \mathrm{~g} . /$ day. On treatment with vitamin $B_{12}$, folic acid, and iron his haemoglobin rose rapidly to $14 \mathrm{~g} . / 100 \mathrm{ml}$. and he was again fully employed except for short periods when he was in hospital with haematuria due to prostatic hypertrophy.
In October 1961 chest $x$-ray films were thought to show evidence of pulmonary tuberculosis and he was given P.A.S. and isoniazid, but he was unable to swallow the cachets. In January 1962 barium swallow showed a mid-oesophageal stricture, and biopsy proved the presence of squamous carcinoma. A tumour dose of 5,000 $\mathrm{r}$ from cobalt -60 was given in 27 days. The immediate response was good, but four months later dysphagia returned and death occurred in July 1962 at the age of 66,13 years after the total gastrectomy. Post-mortem examination revealed a broncho-oesophageal fistula, but with no microscopical evidence of recurrence of growth. There was no sign of active pulmonary tuberculosis and the small intestine was normal.

\section{Case 4}

A married childless woman aged 38 was transferred from Whipps Cross Hospital to the London Hospital in February 1964 for treatment of squamous-cell carcinoma of the mid-oesophagus. She had been thin and dyspeptic all her adult life, with dysphagia since the previous November. Her doctor had given her a course of iron injections in 1960 when she complained of a sore tongue and her haemoglobin had been $9.4 \mathrm{~g} . / 100 \mathrm{ml}$. There was no history of bowel disturbance and she neither smoked nor drank.

She was a thin, pale, freckled woman with a smooth tongue, adequate teeth, and well-shaped but rather brittle nails. Haemoglobin 9.2 g./100 ml., M.C.H.C. $31 \%$, marked macrocytosis, W.B.C. 7,300 (neutrophils $85 \%$, lymphocytes $10 \%$, monocytes $4 \%$, eosinophils $1 \%$ ). Sternal marrow: megaloblastic erythropoiesis. Augmented histamine test meal: free acid present. Normal uptake of vitamin $B_{12}$. Formiminoglutamic acid (F.I.G.L.U.) test: positive. Fasting vitamin-A levels: 28 i.u. $/ 100 \mathrm{ml}$. plasma ; normal uptake of loading dose. Five-day faecal fat excretion $10.5 \mathrm{~g}$./day on a diet containing $50 \mathrm{~g}$. fat daily. On intramuscular iron her haemoglobin rose to $11.8 \mathrm{~g} . / 100 \mathrm{ml}$. in three weeks, but macrocytosis persisted in the peripheral blood until oral folic acid therapy was. started.

She was given a tumour dose of $5,000 \mathrm{r}$ from cobalt-60 in 27 days. The immediate response was satisfactory, but dysphagia recurred after two months, and though partial oesophagogastrectomy was performed in June 1964 local recurrence of growth led to gastropleural fistula, from which the patient died two months later, aged 38. At post-mortem examination the intestinal mucosa appeared normal.

\section{Five-year Survey}

Steatorrhoea was an obvious feature in the first three cases, but in the fourth it might have passed unrecognized except for the presence of macrocytic anaemia.

The possibility that steatorrhoea would sometimes be missed in cases of oesophageal cancer led to an examination of the records of new patients admitted to the London Hospital for treatment of oesophageal cancer during the five-year period 1959-63. This survey was confined to patients suffering from squamous-celled carcinoma of the thoracic oesophagus. There were 41 men and 26 women. Seven of the men had longstanding histories of dyspepsia: two of these underwent gastric surgery for duodenal ulcer 34 and 12 years before development of oesophageal carcinoma, one for gastric ulcer, the interval being four years, and one (Case 3 above) total gastrectomy for pyloric carcinoma 13 years before developing oesophageal carcinoma ; one patient had achalasia of the cardia; and the remaining two had over 20 years' history of unexplained indigestion; one of them aged 62 had been treated for " malabsorption" a few months before the diagnosis of oesophageal cancer. One woman had a 20-year history of gastric ulcer. None of these patients had any gross abnormality in the blood picture at the time of diagnosis of oesophageal carcinoma.

Five female patients had no history of dyspepsia but presented with haemoglobin values of the order of $10 \mathrm{~g} . / 100$ ml., and in two of these the blood film was macrocytic; one was a woman aged 26 who died before the anaemia could be investigated, the other was found to have macrocytic anaemia at the age of 58 , three years after successful irradiation of a mid-oesophageal squamous carcinoma. She did not have 
steatorrhoea, and other tests for absorption were normal. Her anaemia responded fully to folic acid, relapsed when she discontinued the tablets, and responded when she again took folic acid.

The impression gained from examination of the records of the 67 patients with oesophageal cancer was that they were all very sick people and that the pressing need for relief of oesophageal obstruction may have obscured less obvious symptoms. Furthermore, signs of underlying malabsorption states might easily have been attributed to starvation secondary to oesophageal obstruction. In particular, haemoglobin values may have been relatively high owing to dehydration and anaemia therefore missed.

Even when the possibility of malabsorption is considered in these cases, conventional investigation is difficult. It is impossible to perform peroral jejunal biopsy, and failure of patients to ingest a standard diet confuses interpretation of obsorption studies.

\section{Discussion}

More is known about the aetiology of oesophageal cancer than about cancer elsewhere in the gut. Evidence incriminating alcohol is found in the Mortality Tables for 1921, 1931, and 1951 (Registrar-General, 1927, 1938, 1958). Our four cases are of interest in this respect, as none of them had any history of alcoholism. The evidence against tobacco is less convincing (Clemmesen, 1965), but in this context also our patients were relatively abstemious.

In achalasia the incidence of squamous carcinoma is high, but there is little evidence that peptic oesophagitis commonly complicating duodenal ulcer or hiatus hernia is a cause. The growths sometimes associated with hiatus hernia are normally adenocarcinomas and usually have a gastric origin.

The evidence for nutritional deficiency as a factor in oesophageal cancer is strong but non-specific. The curiously high incidence in parts of Africa (Hutt and Burkitt, 1965), part of the Chinese province of Honan, and the island of Curaçao is unexplained (Almy et al., 1964). The high incidence in the Bantu reserve is apparently not related to general malnutrition, but it is obviously difficult to exclude some specific deficiency which affects the oesophageal mucosa.
In the five-year survey of the London Hospital cases of squamous carcinoma of the lower two-thirds of the oesophagus referred to above $12 \%$ of the patients had long-standing dyspepsia. If the single case of achalasia in which oesophageal stasis alone may be important is excluded it would seem that nutritional deficiency could have been significant in one-tenth of the series.

Iron deficiency has long been associated with postcricoid lesions, but the specificity of the relation has been questioned by Elwood et al. (1964). Iron deficiency could be presumed in our cases of steatorrhoea and was clearly present in Case 4, but, in contrast to the Patterson-Kelly sydrome, gross physical signs of iron deficiency were lacking, the anaemia was macrocytic or normocytic, and the growths were situated in the mid-oesophagus in all cases. In the circumstances we feel justified in concluding that, though iron deficiency may have been a factor in the genesis of carcinoma in these cases, some other nutritional deficiency may be more important.

\section{Summary}

Four cases of carcinoma of the thoracic oesophagus occurring in patients with malabsorption syndrome are reported. These cases differ from the Patterson-Kelly syndrome in several respects, and it is suggested that nutritional deficiencies other than iron may be of aetiological importance.

Over $10 \%$ of patients in a series of 67 cases of squamous carcinoma of the thoracic oesophagus had long histories of dyspepsia and possible nutritional deficiency.

Acknowledgements are due to the late Lord Brain for permission to publish Case 2, and we are grateful for Dr. J. Perrin's helpful criticism.

\section{REFERENCES}

Almy, T., et al. (1964). Gut, 5, 196.

Clemmesen, J. (1965). Acta path. microbiol. scand., Suppl. No. 174,

Elwood, P. P. C., Jacobs, A., Pitman, R. G., and Entwistle, C. C. (1964). Lancet, 2, 716.

Hutt, M. S. R., and Burkitt, D. (1965). Brit. med. F., 2, 719.

Registrar-General (1927). Decennial Supplement for 1921: Occupa-

tional Mortality. H.M.S.O., London. 1931 : Occupattonal Mortality. H.M.S.O., London.

(1958). Decennial Supplement for 1951: Occupational Mortality. H.M.S.O., London.
Brit. med. F., 1967, 1, 542-544

Though antibodies to insulin constitute a recognized form of insulin resistance (Lerman, 1944 ; Skom and Talmage, 1958 ; Oakley et al., 1959), all authors agree that insulin antibodies play no significant part in determining insulin dosage in the non-resistant diabetic patient (Moinat, 1958 ; Berson and Yalow, 1959a ; Palumbo et al., 1964a, 1964b). Recent observations from this laboratory (Devlin and Brien, 1965) on the preferential binding of beef, pork, dalanated (de-alanineated) pork, and human insulin by diabetic sera suggested that the role of antibodies in determining insulin dosage was probably more significant than has been hitherto accepted. The present work is an attempt to answer the question of whether antibodies to insulin are a determining factor in insulin dosage in the non-resistant diabetic patient.

\section{MATERIALS AND METHODS}

Forty-two beef-insulin-treated diabetic patients were studied. Sex, age at onset of diabetes, and insulin dosage are given in Table I. The beef preferential binding capacity of the sera with reference to pork, dalanated pork, and human insulin was determined by the method previously described (Devlin and Brien, 1965), with the results shown in Fig. 1. This consists in determining the relative effectiveness of beef, pork, and human insulins to displace beef insulin, 1/125 from the sera tested. The relation between the predicted clinical effectiveness of pork insulin (relative to beef) obtained from the preferential binding-capacity studies and the actual effectiveness of pork insulin in 15 patients who were transferred from beef to pork insulin are shown in Fig. 2. Eight of 